Meta

Journal des traducteurs

Translators' Journal

\title{
La didactique de la traduction médicale, deux ou trois choses que je sais d'elle
}

\section{Christian Balliu}

Volume 50, numéro 1, mars 2005

Enseignement de la traduction dans le monde

Teaching Translation Throughout the World

URI : https://id.erudit.org/iderudit/010658ar

DOI : https://doi.org/10.7202/010658ar

Aller au sommaire du numéro

Éditeur(s)

Les Presses de l'Université de Montréal

ISSN

0026-0452 (imprimé)

1492-1421 (numérique)

Découvrir la revue

Citer cet article

Balliu, C. (2005). La didactique de la traduction médicale, deux ou trois choses que je sais d'elle. Meta, 50(1), 67-77. https://doi.org/10.7202/010658ar
Résumé de l'article

La médecine est une science ancienne, soumise à l'évolution de l'histoire. Son discours, dont la terminologie, ne peut donc échapper à l'influence de ses utilisateurs successifs.

La didactique de la traduction médicale devra donc prendre en compte que le sociolecte médical n'est pas alimenté par une terminologie objective et rigoureuse, mais qu'il est assujetti à une instabilité aussi synchronique que diachronique. 


\title{
La didactique de la traduction médicale, deux ou trois choses que je sais d'elle
}

\author{
CHRISTIAN BALLI U \\ Haute École de Bruxelles, Bruxelles, Belgique \\ cballiu@heb.be
}

\begin{abstract}
RÉSUMÉ
La médecine est une science ancienne, soumise à l'évolution de l'histoire. Son discours, dont la terminologie, ne peut donc échapper à l'influence de ses utilisateurs successifs.

La didactique de la traduction médicale devra donc prendre en compte que le sociolecte médical n'est pas alimenté par une terminologie objective et rigoureuse, mais qu'il est assujetti à une instabilité aussi synchronique que diachronique.
\end{abstract}

\section{ABSTRACT}

An age-old science, medicine has had to follow in the steps of History for millennia. Little wonder, then, if medical language - and, hence, terminology - has always been influenced, over the years, by its successive users.

The didactics of medical translation should take into account that the medical sociolecte, far from feeding on an exact and objective terminology, is in fact prey to synchronic as well as diachronic instability.

\section{MOTS-CLÉS/KEYWORDS}

traduction médicale, didactique, terminologie, objectivité scientifique, sociolecte

\begin{abstract}
On fait la science avec des faits comme une maison avec des pierres.

Mais une accumulation de faits n'est pas plus une science qu'un tas de pierres n'est une maison.
\end{abstract}

Henri Poincaré La Science et l'Hypothèse, 1902.

Personne ne contestera que la traduction médicale, c'est-à-dire celle qui s'attache à des textes de médecine spécialisés écrits par des experts du domaine pour d'autres experts ou, à tout le moins, d'autres médecins, relève d'un enseignement particulier, dissociable de la pédagogie de la traduction générale.

De même, on sait que la différence opérée par les traductologues entre traduction générale et traduction spécialisée repose essentiellement sur le versant lexicologique, la seconde faisant appel à des termes spécialisés, distincts du fonds de la langue commune. C'est ainsi qu'on a pu parler de «langue de spécialité» ou encore de «langues de spécialités» au pluriel, ce qui montre la mouvance de l'expression et des options théoriques qui la sous-tendent.

Je n'entrerai pas ici dans le débat de la langue de spécialité, déjà étudié ailleurs, ni dans cet imbroglio théorique qui consiste à délimiter par on ne sait quelle argutie traductologique la frontière, comme si elle était nette et visible à tous les coups, entre traduction générale et traduction spécialisée. Je ne m’étendrai pas davantage sur les 
ressources terminologiques et documentaires à mettre en œuvre pour garantir l'exactitude dénominative des notions scientifiques qui jalonnent le texte médical. Tout cela est important certes, mais ne relève pas de la traduction proprement dite dans son processus. Il s'agit plutôt de la mise en place d'un système de références et de balises notionnelles dont on aura grand besoin, mais qui ne constitueront qu'un jalon dans le passage du texte de départ au texte d'arrivée. Autrement dit, un texte bien documenté n'est pas forcément un texte bien traduit.

Cette dernière affirmation ne va pas de soi, loin s'en faut. Dans mon expérience d'enseignant, j'ai vu un certain nombre d'étudiants obtenir une note faible en traduction générale et recevoir une note très honorable en traduction spécialisée. La justification donnée par plusieurs collègues et qui peut paraître logique en première analyse est que l'étudiant ne maîtrise pas l'art de traduire, mais qu'il «s'en sort» en traduction spécialisée "parce qu'il s'agit d'étude» et que les termes relatifs au domaine ont été assimilés et rendus dans la traduction. La traduction spécialisée se résumerait par conséquent à l'acquisition d'un vocabulaire spécifique dont la restitution assurerait une traduction correcte. La traduction spécialisée équivaudrait donc à un transcodage terminographique.

Ce postulat fait l'économie de plusieurs éléments essentiels que je me propose précisément d'analyser dans le cadre de cet exposé. Tout d'abord, rendons hommage au vocabulaire de spécialité et voyons-en les atouts et les faiblesses.

\section{Le vocabulaire de spécialité}

C'est volontairement que j'ai utilisé le syntagme vocabulaire de spécialité et non langue(s) de spécialité. Je n'ai qu'une langue maternelle, le français, et c'est dans cette langue que j'exerce mon métier de traducteur. Le français ne se subdivise en aucune manière en "sous-langues", les prétendues langues juridique, informatique, économique, médicale et autres. C'est la raison pour laquelle on entend parler depuis quelques années de discours de spécialité, qui s'émietteraient en discours juridique, informatique, économique, médical, etc. Un peu comme si cette concession faite au langage pardonnait tous les outrages que l'on fait aux textes spécialisés. Car il s'agit bien de textes.

Le texte ne peut se départir de sa composante relationnelle, qui est la raison même pour laquelle il est écrit. Si l'on écrit, c'est pour être lu, un truisme que semblent cependant oublier parfois ceux qui s’intéressent à la littérature spécialisée, à la littérature médicale en particulier. Et la réalité décrite peut être perçue très différemment selon le récepteur. Mieux encore, le texte médical est écrit en fonction du récepteur. Que penser de la phrase suivante, lue il y a quelques années sous la plume d'un cancérologue renommé?

Il est regrettable que le développement de cette magnifique tumeur fût interrompu par le décès du patient.

Une médecine inhumaine? Certainement pas. Déshumanisée? Sans doute. Mais faut-il être expert de l'art de guérir ou grand terminologue devant l'Éternel pour comprendre instantanément que cette phrase ne peut avoir été adressée à un malade ou à ses proches, mais qu'elle a été nécessairement formulée à l'attention d'un confrère? Bien sûr que non. Il est patent que le regret concerne le volet clinique et biologique 
et élude le patient en tant que personnalité morbide. C'est la rançon des progrès scientifiques énormes de la seconde moitié du $\mathrm{xx}^{\mathrm{e}}$ siècle qui voient l'homme comme une succession d'organes et non plus comme un ensemble psycho-physiologique homogène. Cette dénégation du patient peut donner à penser que le vocabulaire médical est obligatoirement aseptisé, dénué de toute connotation et de toute considération "métaphysique», dans le sens où la seule médecine organique a droit de cité. Mais jamais on ne sera tenté de considérer que le médecin se réjouit de la mort d'une personne ni même qu'il s'en désintéresse.

Il me semble aussi remarquable de relever que cette phrase, si elle s'échange d'un expert à l'autre, ne se caractérise pas le moins du monde par un vocabulaire spécialisé. On s'accordera à dire que les adjectifs regrettable et magnifique font partie du vocabulaire courant et même affectif. Le mot tumeur, médical sans conteste, possède des équivalents plus spécialisés, mais néanmoins courants dans la pratique médicale: néoplasie ou dysplasie.

Ce que je veux dire tout simplement, c'est que le médecin ne se départira à aucun moment dans son écriture, qu'il s'agisse d'un protocole, d'observations personnelles ou encore de la publication d'une recherche, de sa condition d'homme, avec son histoire, son éducation et son interaction avec l'environnement socioprofessionnel. La petite phrase que j'ai citée à titre d'exemple prouve à elle seule que la notion de texte médical ne se résume en aucun cas à celle de texte écrit par un médecin, ou encore de texte rédigé par un spécialiste à l'adresse d'un confrère du même domaine de spécialité.

Je crois qu'un texte ne répond pas à une typologie linguistique bien établie, fondée sur des moyens lexicaux, terminologiques et stylistiques, mais s'appuie sur des référents notionnels qu'il convient de bien cerner et qui s'expriment par des biais très différents selon les acteurs en présence (l'auteur et le récepteur) et le support du texte.

Je m'explique. Si le texte est écrit dans une des revues médicales de pointe, ce support va conditionner - le mot n'est pas trop fort - l'emballage linguistique et stylistique du contenu.

La langue tout d'abord. On sait que depuis la Seconde Guerre mondiale, la médecine et la recherche médicale sont marquées par la civilisation anglo-saxonne, pour des raisons économiques essentiellement. Il est donc normal que les grandes revues médicales, généralistes ou spécialisées, appartiennent à ce monde anglosaxon. Je pense à The Lancet, au New England Journal of Medicine (NEJM), au British Medical Journal ou au Journal of the American Medical Association (JAMA). Cela explique que nombre d'auteurs, français, espagnols, allemands et autres, publient directement dans une langue qui n'est pas leur langue maternelle, qui est tout au plus leur langue de travail.

Le style ensuite. Leur maniement de l'anglais reste dès lors approximatif, mécanique, et ne leur permet pas d'exprimer leur pensée dans toutes ses nuances. Leur style en devient plus impersonnel, non parce que le style médical est ainsi fait comme on l'a trop souvent affirmé, mais parce que la carence expressive se manifeste par une absence de connotation, par une espèce d'infirmité affective. La neutralité d'un certain lexique scientifique permet à ces chercheurs de dissimuler leur incompétence linguistique. En d'autres mots, on préfère s'exprimer moins bien pour être lu davantage. Une véritable aporie scientifique. Mais la course à la notoriété, aux crédits pour alimenter la recherche, fait que la quête de publicité le dispute à la nécessité vitale. 
Cette notion - très anglo-saxonne - du publish or perish n'est pas sans incidence sur l'évolution du langage médical, une incidence plutôt négative on en conviendra.

La conjugaison des deux éléments que je viens de décrire ne peut qu'engendrer un style, et même un lexique, de plus en plus approximatifs. La carence expressive se double d'une nécessité d'uniformisation, afin que la communauté scientifique tienne le même langage: écrire de la même manière montre que l'on appartient à la même élite scientifique. Partager les modes d'expression, c'est partager les compétences et la renommée. C'est ce que j'appelle «la force des mots».

Malheureusement, la force des mots se mue souvent insidieusement en «farce des mots». Comme on participe de la même communauté, des mêmes rites, des mêmes préoccupations scientifiques, on parle de la même chose et ce même, si la précision énonciative diffère. Au fond, qu'importe l'exactitude de la formulation, puisque tout le monde sait de quoi il retourne et que la connaissance pointue du domaine corrigera aussitôt l'expression défaillante.

Danica Seleskovitch ne disait pas autre chose à propos de l'interprétation: quand l'interprète connaît le sujet, quand il peut mobiliser son bagage cognitif, il évolue en terrain sûr. Elle m'avait confié un jour qu'elle se sentait le plus vulnérable lorsqu'elle ne connaissait pas le domaine abordé par un orateur. Il serait erroné de croire que le bagage affectif quant à lui ne concerne que l'interprétation et non les textes spécialisés; les conférences sont des «textes» comme les autres, des discours comme il existe des «discours» médicaux. D’ailleurs, les conférences peuvent être très techniques, mais le conférencier ajoutera toujours à la somme des signes linguistiques sa touche personnelle, son propre viatique affectif. C'est parce que l'affectif est omniprésent, y compris dans les textes de médecine, que la monoréférentialité ou, si l'on préfère, la biunivocité ne sont pas la règle. Un élément qui devrait intéresser les terminologues dans les années à venir et réorienter la discipline vers un cadre textuel plus large que le terme, la notion ou même l'idiomaticité. La terminologie n'a pas encore réussi à ce jour à s'extraire de sa gaine linguistique. Je persiste à croire que la philosophie du langage a un rôle très important à jouer dans la mise en évidence et l'analyse des sociolectes.

\section{Le sociolecte médical}

Il existe une littérature médicale, à savoir une forme d'écriture propre à la médecine et aux médecins. Cette littérature professionnelle renvoie par conséquent à un métier, lequel existe de manière organisée depuis la Grèce antique et Hippocrate. Auparavant, la médecine, notamment égyptienne mais aussi assyrienne, était magique et incantatoire, exercée par des devins ou des personnages semi-légendaires comme Imhotep, son dieu égyptien.

L'exercice de la médecine plonge donc ses racines dans l'histoire ancienne et est tributaire de l'évolution des sociétés; la médecine fait à son tour évoluer ces dernières. L'histoire des sociétés marque à coup sûr la nomenclature scientifique et, notamment, médicale. Un exemple: Hippocrate appelle l'épilepsie «maladie sacrée», en raison du fait que son apparition soudaine et apparemment inexpliquée était due, selon les adeptes de la médecine magique, à une force surnaturelle, divine en l'occurrence. Dans le même temps, il lutte contre cette appellation en déclarant au début du Traité qu'il consacre à cette maladie: 
La maladie sacrée ou épilepsie n'est pas plus sacrée que les autres maladies.1.

Les croyances, les hypothèses et, plus tard, les théories dignes de ce nom influenceront les dénominations scientifiques. Dégager la médecine de son évolution, de son histoire pour étudier sa terminologie in abstracto, c'est courir le risque de mal interpréter les dénominations, de croire ingénument que la formation strictement linguistique du terme - par radicaux et dérivés - en explique et en éclaire le sens. C'est croire aussi à la formation uniquement scientifique des termes, alors que les racines - le mot même le dit - ont subi des avatars qui font que la dénomination ne recouvre pas nécessairement la notion envisagée. C'est le voyage des mots qui induit un décalage, parfois important, entre le terme et la notion.

Mais voyons les choses de manière plus concrète. Les mots anémique et exsangue signifient étymologiquement la même chose, la seule différence au départ étant que le premier est formé sur une racine grecque, tandis que le second est formé sur une racine latine. Ils ne sont aujourd'hui en aucune façon synonymes, le premier indiquant un sujet atteint d'érythrocytopénie (une carence en globules rouges) et le second indiquant un sujet qui a perdu tout son sang. Notons aussi que le premier, anémique, est un terme, dans la mesure où il s'utilise uniquement dans un contexte médical. Le second est un mot, rien de plus - je devrais dire rien de moins -, fait partie du vocabulaire courant et est utilisé en dehors d'un contexte médical stricto sensu.

Que dire des mots composés à la fois d'une racine latine et d'une racine grecque? Est-ce là une méthode scientifique d'assurer la composition des termes savants? Non, évidemment. Et pourtant ils sont légion. Serge Quérin a colligé environ 250 hybrides gréco-latins et a relevé que:

une dizaine sont en concurrence avec un synonyme étymologiquement homogène; la moitié au moins connaissent un emploi quelque peu différent de leur "équivalent» plus "pur», alors que les autres n'ont pas d'équivalent usité de la sorte 2 .

Pour donner un exemple, l'hybride endoveineux se dit généralement d'un dispositif placé à demeure dans une veine, comme une électrode, ou de ce qui concerne l'endoveine. Par contre, l'homogène intraveineux s'utilise à propos d'une injection ou d'une substance administrée dans une veine, ou encore de ce qui est relatif à l'intérieur d'une veine.

Un cas amusant de concurrence curieuse entre hybrides et homogènes est le cas des médications dirigées contre les infections à champignons. Le composé latin homogène est fongicide, le composé grec homogène étant antimycosique. Il existe aussi un hybride, antifongique. Les trois sont synonymes parfaits, de même que fébriguge et antipyrétique ou encore vomitif et émétique. Il est vrai que l'étymologie latine, notamment dans le cas d'un hybride, favorise la compréhension du public francophone; cancérologie est sans doute plus transparent que oncologie, pourtant formé de manière homogène.

On peut donc déjà déduire de ce qui précède que la formation des termes de médecine ne suit pas une logique rigoureuse, moins encore linguistique. Il serait illusoire de vouloir bannir ces hybrides du vocabulaire médical au nom d'un purisme terminologique de mauvais aloi, le problème des équivalences demeurant entier. En effet, les errances séculaires de la médecine ont forgé une terminologie parfois erratique, qui tantôt a disparu sous les coups de butoir du progrès, tantôt s'est maintenue contre vents et marées, en dépit de son inexactitude. Cela me rappelle le diagnostic 
amer de François Magendie, l'initiateur de la physiopathologie au xıx $x^{\mathrm{e}}$ siècle, qui lutta contre le vitalisme tenace de son époque et déclarait dès 1809:

Si l'on chassait les médecins de l'Hôtel-Dieu, la mortalité serait peut-être moindre

À cet égard, je citerai un exemple dont tout le monde a entendu parler. Après les événements du 11 septembre 2001, le terme médical le plus utilisé par les médias pendant de longues semaines a été sans conteste le mot anthrax. Ce mot français désigne à la fois la forme aiguë et bénigne de la maladie. Utilisé en l'espèce pour désigner la forme aiguë, le terme a bien entendu un équivalent espagnol qui est carbunco $^{4}$. Mais un homonyme et quasi-synonyme du terme français existe aussi dans la langue de Cervantès : ántrax. Ce dernier terme désigne la forme bénigne de la maladie et donc une réalité nosologique et épidémiologique tout à fait différente ${ }^{5}$. Ce chiasme entre la terminologie française et espagnole ne peut que laisser le lecteur et le traducteur médical perplexes. Il prouve une fois de plus que la terminologie médicale est labile et instable, non uniforme dans les différentes cultures.

Une autre réflexion qu'il me semble intéressant de faire à ce stade porte sur le sociolecte en tant qu'appropriation d'une terminologie par les praticiens, dont seraient exclus ceux qui ne sont pas membres de la communauté scientifique. On touche ici d'une certaine manière au souci performatif du jargon médical. J'ai déjà souligné ailleurs la personnalisation du discours, le rôle de la connotation ou le recours à l'éponymie ${ }^{6}$. Je n'y reviendrai pas ici.

Il y a indubitablement une notion de caste qui traverse l'histoire de la discipline. En français, le titre de Docteur que l'on utilise lorsqu'on s'adresse au médecin est réservé à lui seul, alors que nombre d'autres professions requièrent l'obtention d'un doctorat pour être exercées. On y sent nettement l'influence de la Faculté et la volonté de réserver cet honneur à une élite. Et comme une élite se doit de posséder un langage à l'aune de sa situation...

Le caractère élitaire de la profession se matérialise déjà au Moyen Âge, quand le médecin lisait - toujours la «force des mots» - la leçon d'anatomie à partir de sa chaire (d'où il professait ex cathedra), sans recourir encore à un barbier, ce factotum de la médecine, chargé de découper les corps dès la Renaissance. Il y avait à l'époque une dichotomie entre la parole, celle des grands textes fondateurs d'Hippocrate ou de Galien, et le geste. Comme je viens de le signaler, ce n'est qu'à partir de la Renaissance que l'on joindra le geste à la parole et que le logos sera vérifié, souvent infirmé, par une vérification in situ. Dans Le Malade imaginaire, Molière mettra en scène de façon remarquable et savoureuse ces médecins dont la vanité linguistique tient lieu de compétence médicale. De façon identique, le terme scientifique tient parfois lieu de diagnostic, alors qu'il ne fait que traduire de manière plus absconse la description faite par le patient à l'aide de simples mots. Un érythème remplacera dans la bouche du médecin le mot rougeur et une hypotension orthostatique sera le pseudo-diagnostic asséné à un malade qui rapporte des vertiges au moment de se lever. Le vocabulaire savant fait prendre une description, un symptôme pour un diagnostic. La confusion entre séméiologie et diagnostic est de la sorte savamment entretenue.

Aujourd'hui encore, le discours médical reste volontairement hermétique, pour des raisons essentiellement liées à l'hyperspécialisation croissante de la discipline. Chaque tiroir de la discipline essaie effectivement de créer son propre vocabulaire, nourri en permanence par l'évolution du domaine en question. Le mot microbe date 
ainsi de la deuxième moitié du $\mathrm{XIX}^{\mathrm{e}}$ siècle et fut créé par Émile Littré, plus connu pour son apport à la langue française que pour sa carrière de médecin. Dans le même ordre d'idées, il me paraît indéniable que Bichat a inauguré à la fin du XVIII siècle une nouvelle conception d'ensemble de la médecine qui a transpiré sur la terminologie en vigueur. Ainsi, Bichat, fondateur de l'anatomie générale et apôtre de l'histologie, déclare comme un coup de tonnerre dans un ciel serein:

C'est parce que l'homme est mortel qu'il est malade et non pas parce qu'il est malade qu'il meurt ${ }^{7}$.

Ce stockage permanent de vocabulaire est assez typique de la médecine, l'informatique étant une science extrêmement jeune où la dimension diachronique est inexistante. Le droit pour sa part, qui a aussi une tradition plurimillénaire, évolue moins rapidement et est beaucoup plus marqué par la tradition et un certain conservatisme. Ce foisonnement de la terminologie médicale et la multiplication des tiroirs engendrent un emmagasinage constant de données et de notions.

Il y a par conséquent une alchimie entre acquis diachroniques, le noyau dur de la discipline, et nouveautés synchroniques, que le traducteur médical ne maîtrisera que difficilement. C'est pourquoi je n'ai de cesse de plaider pour la collaboration constante avec les praticiens qui constituent sans doute aucun les meilleurs fournisseurs de bases de données terminologiques. Ils allient en effet connaissance approfondie de l'art de guérir (les bases) et pratique professionnelle quotidienne (les progrès). Ce que je veux dire, c'est que ce sont les dictionnaires et les encyclopédies médicales (comme les Principles of Internal Medicine de Harrison) qui fournissent les fondements, les informateurs se chargeant de l'actualisation, point faible des dictionnaires. Ce passage obligé par le sociolecte médical constitue le tremplin idéal vers le point suivant: les mots au secours des termes.

\section{Les mots au secours des termes}

Un élément me paraît peu considéré lorsque l'on traite de traduction médicale: c'est le rôle des mots. Un peu comme si ceux-ci ne servaient qu'à remplir les creux lexicaux qui séparent les termes et les notions, et n'étaient que des charnières. Les mots ne sont pas que des chevilles ou des connecteurs. Ils alimentent la dialectique du texte en enchevêtrant les notions et les unités notionnelles dans l'écheveau complexe de l'hyperonymie et de l'hyponymie d'une part, et de la coordination et de la subordination d'autre part. Les mots relient les termes et leur confèrent un sens dynamique.

L'hyperonymie et l'hyponymie sont déjà un procédé d'enchâssement des notions qui se fait au niveau lexical. La lexicalisation des notions est d'ailleurs une des faiblesses actuelles de la terminologie. Le mythe de la terminologie, c'est la nécessité absolue de trouver l'équivalent lexical exact, ce qui focalise l'attention de l'étudiant sur le mot juste - qui devient dès lors le «terme» - et lui fait omettre ce que Vinay et Darbelnet appelaient les stades II et III, à savoir les niveaux syntaxique et textuel ${ }^{8}$. La terminologie est, dans un nombre non négligeable de cas, la loupe qui met en avant l'arbre qui cache la forêt.

Or, le terme fait partie d'un ensemble qui va au-delà du giron étroit de la phrase ou du paragraphe pour se fondre dans un système sémantique beaucoup plus large, matérialisé par le texte dans son ensemble et imposé par l'auteur en particulier. D’où 
la tendance de la terminologie contemporaine à envisager le cadre plus large de la phraséologie.

Précisément, le texte dans son intégralité constitue un vaste réseau de superordination et de subordination des notions et agit en conséquence à un niveau plus élevé que celui des termes. Les mots prennent alors le relais et assurent la connexion des termes qui ne fonctionnent plus en autarcie, de manière autonyme, mais qui se voient assigner une fonction sémantique dans un cadre plus large, celui du sens global.

Prenons un court extrait du livre Des virus et des hommes, écrit par le professeur Luc Montagnier de l'Institut Pasteur:

Pourquoi du sang? Pourquoi des ganglions? On sait à l'époque que le SIDA est une maladie qui détruit les lymphocytes T4 et qui est précédée de gonflements ganglionnaires qui peuvent persister des mois, voire des années. Il est donc logique pour moi de penser que l'agent $d u$ SIDA se trouve non seulement dans le sang, mais aussi dans les ganglions lymphatiques. Ces derniers sont en effet, avec la rate, le réservoir par excellence des lymphocytes. Chaque ganglion constitue un point d'arrêt pour les germes envahisseurs, virus ou bactéries. Les lymphocytes proliferent alors et le ganglion augmente de volume, pour régresser quand l'infection est jugulée. S'il persiste, c'est au contraire le signe que l'infection devient chronique ou que l'inflammation a une autre origine, par exemple cancéreuses.

Ce texte est très instructif. Ce sont les termes qui tissent la trame du domaine, en l'espèce celui de l'immunologie appliquée; le nom de l'auteur fournit aussi cette information. Mais ce sont les mots qui mettent le décor en place, qui situent les termes les uns par rapport aux autres dans des relations de préséance, de sujétion, de conséquence, de concession, de temporalité ou encore de finalité. Le verbe détruire montre que le HIV est lymphotrope, le verbe précéder indique que le stade IV est consécutif à la polyadénopathie, le verbe régresser signale la lymphopénie lorsque le système immunitaire abandonne la lutte, et je pourrais allonger la liste.

En montrant de la sorte la fonction dévolue aux mots, j’ai par la même occasion prouvé que je pouvais utiliser une terminologie encore plus spécialisée que celle employée par Montagnier lui-même. Le texte en aurait été plus hermétique, plus cryptique, du moins pour le commun des mortels, mais en aurait-il été pour autant plus pointu? Absolument pas. Le recours aux termes n'est donc pas un garant de la spécialisation d'un domaine; il n'est pas non plus indispensable pour que le texte soit considéré comme spécialisé. La condition n’est ni nécessaire ni suffisante. Quelle différence y a-t-il par exemple entre ces deux assertions?

- Le virus du SIDA s'attaque aux lymphocytes, ce qui induit une diminution sensible des cellules T4.

- Le HIV est lymphotrope, ce qui induit une lymphopénie T4.

Il n'y a aucune différence sémantique; la seule chose que l'on puisse dire est que la première assertion est plus accessible au grand public et que la seconde obéit aux lois de l'économie linguistique. Le désavantage de l'hermétisme terminologique est compensé par une concision de l'énoncé, pas nécessairement par une précision accrue. Je dirais même que le verbe s'attaquer de la première version est plus précis car il montre le caractère agressif, pathogène, du virus, ce que ne rend pas l'adjectif lymphotrope de la seconde version. Le tropisme d'un antigène ou d'une substance, médicamenteuse par exemple, est son attirance, sa prédilection pour une région particulière. Ainsi, un psychotrope est une substance agissant sur le psychisme (un antidépresseur 
ou un tranquillisant), mais ne l'attaquant pas, que du contraire. Une fois encore, l'imprécision terminologique est claire, et c'est le mot s'attaquer qui est venu au secours du terme, car la connotation qu'il ajoute est éclairante: la première phrase implique une morbidité, ce que la seconde ne laisse pas entrevoir.

Voilà la clef. Un texte médical ne peut faire l'économie du fait qu'en fin de compte, il concerne toujours l'homme, dans ce qu'il a de plus humain et de plus fragile, la vie. Pour cette raison, le texte médical est presque toujours connoté, parfois de manière nette par un recours patent aux mots, parfois de manière plus latente, mais non moins réelle. La connotation d'un texte médical ne se mesure pas mathématiquement, par addition de termes ou soustraction de mots. La linguistique mathématique ne rendra jamais compte du degré de dénotation et de connotation d'un texte médical et la connaissance des notions sous-jacentes ne constituent qu'une étape de la compréhension du texte.

Je donnerai un deuxième exemple que j'emprunte à Jean Hamburger qui dirigea, à l'hôpital Necker, un ensemble d'unités de soins, d'enseignement et de recherches ${ }^{10}$, consacré aux fonctions, maladies et transplantation du rein:

Un homme de cinquante ans éprouve de la gêne et des douleurs à la marche et son médecin découvre que ses vertèbres et son bassin commencent à être atteints d'une maladie rhumatismale qu'on nomme spondylarthrite ankylosante. Or, bien que sa santé fût jusqu'alors parfaite, cet homme était presque sûrement marqué dès son origine, puisque la plupart de ces malades (plus de $90 \%$ d'entre eux) ont sur leurs cellules un marqueur héréditaire HLA désigné par l'étiquette B27, qu'on ne retrouve que chez $5 \%$ des autres hommes. De même, la lèpre, l'allergie au pollen, le psoriasis, la myasthénie, et bien d'autres maladies semblent favorisées par certains marqueurs ou couples de marqueurs $H L A^{11}$.

Le texte et sa signature ne laissent aucune place au doute. Encore un texte d'immunologie appliquée écrit par l'un des plus grands spécialistes de la seconde moitié du $\mathrm{xx}^{\mathrm{e}}$ siècle. Il ne faudrait pas croire que ce sont les termes qui permettront au traducteur de circonscrire et de comprendre le domaine dont il est question. Dès la première phrase, la mention de la spondylarthrite ankylosante évoque les maladies autoimmunes, non par son contenu terminologique, mais plutôt par son environnement contextuel, dont le signataire du texte.

L'âge du début de l'affection, sa rareté, le rapport au HLA (Human Leucocyte Antigen), à savoir au CMH (complexe majeur d'histocompatibilité) exclusivement humain, la référence au locus B27 et le lien avec le psoriasis notamment, circonscrivent sans doute aucun les affections auto-immunes. On peut même pousser l'analyse plus avant: la connaissance de la trajectoire de Jean Hamburger, l'utilisation du verbe sembler dans la dernière phrase et la liaison avec la lèpre ou la myasthénie indiquent que l'extrait a dû être écrit dans les années 1970. En effet, l'immunologie ne prend son essor dans les études médicales qu'à partir de 1960; d'autre part, l'étude du SIDA à partir du début des années 1980 a favorisé une meilleure appréhension des maladies auto-immunes, parmi lesquelles on range aujourd'hui le psoriasis et la sclérose en plaques, mais en aucune manière la lèpre $\mathrm{e}^{12}$ par exemple. Tout cela montre combien la médecine évolue rapidement et combien les banques de données terminologiques peinent à suivre le rythme imposé par les progrès médicaux.

Comme chez Montagnier, le texte regorge d'imprécisions scientifiques (de la gêne et des douleurs, qu'on nomme, était presque sûrement marqué, la plupart de ces malades, bien d'autres maladies, semblent être favorisées, certains marqueurs) et comporte des 
mots, voire des syntagmes non marqués objectivement (un homme de cinquante ans, éprouve, santé jusqu'alors parfaite). En réalité, le texte concerne moins une maladie auto-immune qu'un patient qui souffre. Ce sont les mots qui renferment l'essence du message et les termes n'existent que par eux.

Comprendre le texte, c'est précisément réhabiliter le mot, dans ce qu'il a de plus riche, à savoir la nuance, le coloris. C'est aussi savoir le corriger quand il est imprécis, même si, comme je crois l'avoir montré, il n'est pas plus imprécis que le terme. Voici un exemple où la terminologie n'aide pas à corriger l'imprécision, mais où la connaissance du domaine est précieuse. Le texte est pourtant écrit par un expert du métabolisme des lipides:

Rappelons que les phénotypes athérogènes sont les types II et III. Chez les patients dont la cholestérolémie est augmentée, il faut établir si l'hypercholestérolémie résulte d'une augmentation des LDL. Il est en effet bien établi actuellement que la diminution d'une telle hypercholestérolémie peut réduire, même si l'élévation du cholestérol est modeste, le risque de coronaropathie. Par ailleurs, il est raisonnable de faire chuter une hypertriglycéridémie par une modification des habitudes alimentaires, bien que l'on ne sache pas actuellement si ceci améliore le risque de coronaropathie. Il se pourrait que le risque associé à un LDLChol/HDL Chol élevé, soit modulé par les $T G^{13}$.

On aura beau avoir recherché les termes phénotypes, hypercholestérolémie et hypertriglycéridémie, on n'aura pas pour la cause traduit convenablement le texte. La résolution des problèmes terminologiques et l'éclaircissement des notions ne donnent pas une vue d'ensemble du texte; la connaissance générale de la discipline permet de détecter d'éventuelles erreurs dans l'articulation syntaxique ou des lacunes d'écriture qui peuvent fourvoyer le profane. Dans l'extrait cité, il est évident qu'une chute du taux de triglycérides va faire diminuer le risque d'accidents cardiovasculaires et que le choix par l'auteur du verbe améliorer pourrait laisser penser exactement l'inverse. Mais pour le spécialiste, il n'y aura aucun doute, sa compétence et sa pratique quotidienne suppléant au relâchement de l'écriture.

Il n'existe pas de concordance entre terminologie et domaine de spécialité, tout comme ce dernier ne sacrifie pas la langue générale sur l'autel d'une dénotation omniprésente. Le discours de spécialité n'est que l'actualisation d'un pan de la langue générale, à des fins techniques ou scientifiques. L'idée d'un texte où l'auteur ferait l'économie de sa personnalité dans l'écriture semble peu convaincante, a fortiori dans un texte scientifique où la part d'investissement du chercheur dans l'exposé des motifs est prépondérante.

C'est la raison pour laquelle l'éclairage du domaine ne doit en aucune façon se résumer à l'étude du texte fourni aux étudiants. Le texte n'est qu'un prétexte pour aborder l'histoire de la discipline, sa résonance sur la terminologie qu'il véhicule et la mise en perspective diachronique des notions. Avoir deux paragraphes d'avance sur l'étudiant dans sa préparation n'est qu'un mirage; c'est l'hôpital qui se moque de la charité.

Le terme est plus faible que le mot, sa limite est synchronique et son exactitude parfois illusoire. Les dictionnaires médicaux, comme les autres, sont dans la classification proposée des dictionnaires de signifiants. La démarche gagnerait à être inversée : remonter du signifié au signifiant signifie partir du référent vers sa représentation linguistique. La correspondance serait de la sorte évincée au profit de l'équivalence qui ne confond pas emballage lexical et contenu textuel. 
La traduction médicale, qui enchevêtre notions scientifiques et approche humaine, n'échappe pas à la règle. Les exemples cités montrent que ce sont les mots, issus de la langue générale, qui tissent la toile cognitive du texte et donnent leur sens aux termes, dont l'autarcie sémantique n'est qu'un trompe-l'œil.

\section{NOTES}

1. De la maladie sacrée, dans Hippocrate de Cos, De l'art médical, traduction d'Émile Littré, Paris, Le Livre de Poche, 1994, p. 126.

2. Serge Quérin, «Emploi de termes hybrides gréco-latins dans le langage médical», traduction médicale et documentation, Meta, Montréal, mars 2001, vol. 46, $\mathrm{n}^{\circ}$ 1, p. 7.

3. Voir E. Ackerknecht, La médecine hospitalière à Paris (1794-1848), Paris, Payot, 1986; de même, J. Olmsted, François Magendie, New York, Schuman's, 1944.

4. Le mot carbunco se rencontre déjà chez Isidore de Séville au vi ${ }^{e}$ siècle. Ce dernier Père de l'Église d'Occident écrivit l'encyclopédie Étymologies, appelée aussi Origines, somme du savoir profane et religieux de l'époque.

5. Notre anthrax (carbunco en espagnol) est causé par le Bacillus anthracis, alors que l'ántrax espagnol est provoqué par des staphylocoques.

6. À ce propos, on consultera: Christian Balliu, «L’enseignement de la traduction médicale: pour une nouvelle pragmatique», La traduction et l'interprétation dans la Belgique multilingue, Meta, Montréal, mars 1994, vol. 39, n 1, p. 15-26. Du même auteur: "Les traducteurs: ces médecins légistes du texte», traduction médicale et documentation, Meta, Montréal, mars 2001, vol. 46, nº 1, p. 92-102.

7. Xavier Bichat, Lettre à Pinel, Paris, 1803 (éditée un an après sa mort).

8. Jean-Paul Vinay et Jean Darbelnet, Stylistique comparée du français et de l'anglais, $1^{\text {re }}$ éd. 1958, Paris, Didier, 1972, p. 45-46.

9. Luc Montagnier, Des virus et des hommes, Paris, éd. Odile Jacob, 1994, p. 49.

10. Jean Hamburger avait compris que la recherche, tout comme l'enseignement, est indissolublement liée à la pratique du terrain.

11. Jean Hamburger, L'Homme et les hommes, Paris, Flammarion, 1976, p. 26-27.

12. Cette maladie est causée par le bacille de Yersin.

13. Yves Mardens, Troubles du métabolisme des lipides, Université libre de Bruxelles, 2000. 